\title{
artigo
}

Júnior, A. R.C., Martins, M. I. S., Alcântara, D. G., Santos, M. A. P., Teixeira, A. N. A., Gomes, T. F., Dores, C. C. C., Freitas, F. A. S., Porto, A. P. M., Moreira, F. J. F.. Investigação clínica e sintomatologica de pacientes acometidos pela covid-19 entre 2020 e 2021 no Ceará

\section{Investigação clínica e sintomatologica de pacientes acometidos pela covid-19 entre 2020 e 2021 no} Ceará

\author{
Clinical and symptomatological investigation of patients affected by covid-19 between 2020 and 2021 in Ceará \\ Investigación clínica y sintomalógica de pacientes afectados por covid-19 entre 2020 y 2021 en Ceará
}

\begin{abstract}
RESUMO
Objetivo: descrever o comportamento clínico e sintomatológico de pacientes acometidos pela COVID-19 no Ceará. Métodos: Estudo descritivo, retrospectivo, de análise documental com abordagem quantitativa, realizado em 3 hospitais de referência para tratamento da COVID-19 no Ceará. Incluídos 965 pacientes divididos entre primeira e segunda onda, conforme data de internação. Os dados foram obtidos através da plataforma "ResCOVID". Resultados: O perfil descreve de forma respectiva $1^{\text {a }}$ e $2^{\mathrm{a}}$ onda, apresentando idade entre 50 a 59 anos 26 (16,9\%) e 140 (17\%), sexo masculino $89(57,8 \%)$ e 454 (56\%). Hipertensão $75(48,7 \%)$ e $364(44,9)$; diabetes $38(26,7 \%)$ e $222(27,4)$ foram as comorbidades mais frequentes. Os sintomas mais reportados foram dispneia $112(72,7 \%)$ e 610 (75,2\%); tosse $108(70 \%)$ e 491 (55,7\%); febre 104(67,5\%) e 443 (50,2\%). Conclusão: Foi possivel realizar a caracterização da população, evidenciando variaçães no perfil clínico e sintomatológico e diminuição em número de óbitos na segunda onda.
\end{abstract}

DESCRITORES: Infecções por coronavírus; Pandemias; Organização Mundial de Saúde; Epidemiologia; Avaliação de Sintomas.

\section{ABSTRACT}

Objective: to describe the clinical and symptomatological behavior of patients affected by COVID-19 in Ceará. Methods: Descriptive, retrospective study of document analysis with a quantitative approach, carried out in 3 reference hospitals for the treatment of COVID-19 in Ceará. 965 patients were included, divided between the first and second wave, according to the date of admission. Data were obtained through the "ResCOVID" platform. Results: The profile describes the 1st and 2nd wave respectively, aged between 50 and 59 years old, $26(16.9 \%)$ and 140 (17\%), male 89 (57.8\%) and 454 (56\%). Hypertension $75(48.7 \%)$ and 364 (44.9); diabetes $38(26.7 \%)$ and $222(27.4)$ were the most frequent comorbidities. The most reported symptoms were dyspnea $112(72.7 \%)$ and $610(75.2 \%)$; cough $108(70 \%)$ and $491(55.7 \%)$; fever $104(67.5 \%)$ and $443(50.2 \%)$. Conclusion: It was possible to characterize the population, showing variations in the clinical and symptomatological profile and a decrease in the number of deaths in the second wave.

DESCRIPTORS: Coronavirus Infections; Pandemics; World Health Organization; Epidemiology; Symptom Assessment.

\section{RESUMEN}

OBJETIVO: describir el comportamiento clínico y sintomatológico de pacientes afectados por COVID-19 en Ceará. Métodos: Estudio descriptivo, retrospectivo de análisis de documentos con abordaje cuantitativo, realizado en 3 hospitales de referencia para el tratamiento de COVID-19 en Ceará. Se incluyeron 965 pacientes, divididos entre la primera y la segunda oleada, según la fecha de ingreso. Los datos se obtuvieron a través de la plataforma "ResCOVID". Resultados: El perfil describe la $1^{\text {a }}$ y $2^{\text {a }}$ oleada respectivamente, con edades comprendidas entre 50 y 59 años, 26 (16,9\%) y $140(17 \%)$, hombres $89(57,8 \%)$ y $454(56 \%)$. Hipertensión $75(48,7 \%)$ y $364(44,9)$; La diabetes $38(26,7 \%)$ y $222(27,4)$ fueron las comorbilidades más frecuentes. Los síntomas más reportados fueron disnea $112(72,7 \%)$ y $610(75,2 \%)$; tos 108 (70\%) y 491 (55,7\%); fiebre $104(67,5 \%)$ y 443 (50,2\%). Conclusión: Fue posible caracterizar la población, mostrando variaciones en el perfil clínico y sintomatológico y una disminución en el número de muertes en la segunda ola.

DESCRIPTORES: Infecciones por Coronavirus; Pandemias; Organización Mundial de la Salud; Epidemiología; Evaluación de síntomas.

RECEBIDO EM: 21/10/2021 APROVADO EM: 10/12/2021

\section{André Ribeiro de Castro Júnior}

Enfermeiro pela Universidade Estadual do Ceará - UECE. Mestre em Cuidados Clínicos em Enfermagem e Saúde pela Universidade Estadual do Ceará. Doutorando em enfermagem, com ênfase na Promoção da Saúde, pela Universidade Federal do Ceará.

ORCID: 0000-0002-3681-3607 


\section{Maria lara Socorro Martins}

Fisioterapeuta pela Faculdades Nordeste/ DeVry, especialista em psicomotricidade. Doutoranda em Saúde Pública pela Universidade Federal do Ceará.

ORCID: 0000-0001-9366-8621

\section{Daniel Germano Alcântara}

Fisioterapeuta pela Faculdades Nordeste/ DeVry. Especialista Saúde da Família pela UNILAB. Fisioterapeuta do Hospital Ana Lima (Hapvida).

ORCID: 0000-0002-0798-6293

\section{Marcos Augusto de Paula Santos}

Enfermeiro pela Universidade Católica do Salvador, (UCSal), 2019 Brasil. Pós- Graduação em Assistência em Oncologia e Onco-Hematologia com Ênfase na Multidisciplinaridade pela Universidade Salvador (UNIFACS).

ORCID: 0000- 0003-3632-3904

\section{Ana Naiara Alves Teixeira}

Enfermeira pela Universidade de Fortaleza - UNIFOR (2015). Especialização em Saúde Pública pela Escola de Saúde Pública do Ceará.

ORCID: 0000-0002-4069-0515

\section{Ticiane Freire Gomes}

Graduada em Enfermagem pela Universidade de Fortaleza - UNIFOR. Pós-graduada em Auditoria, gestão e perícias em sistemas de saúde pela Universidade Estadual do Ceará. Mestre em Enfermagem na Universidade da Integração Internacional da Lusofonia Afro-Brasileira-UNILAB.

ORCID: 0000-0002-8469-1472

\section{Camila Campos Colares das Dores}

Cientista de Dados. Doutoranda pelo Programa de Pós- Graduação em Ciência da Computação da Universidade Estadual do Ceará - UECE.

ORCID: 0000-0002-2619-665X

\section{Francisco Aislan da Silva Freitas}

Cientista de Dados. Doutoranda pelo Programa de Pós- Graduação em Ciência da Computação da Universidade Estadual do Ceará - UECE.

ORCID: 0000-0002-0949-1876

\section{Ana Paula Matos Porto}

Médica Infectologista. Doutoranda pelo Programa de Pós- Graduação de Doenças Infecciosas e Parasitárias da Faculdade de Medicina da Universidadede São Paulo - FM USP.

ORCID: 0000-0003-3641-9746

\section{Francisco Jadson Franco Moreira}

Psicólogo. Doutorando em Educação - Universidade Estadual do Ceará (UECE). Coordenador do Centro de Investigação Cientifica da Escola de Saúde Pública do Ceará.

ORCID: 0000- 0003-3141-4700

\section{INTRODUÇÃO}

A pós divulgação do surgimento em dezembro de 2019, o novo coronavírus, responsável pela infecção reconhecida como COVID-19, surge associado com diversos sintomas e amplo espectro de complicações. As formas prevalentes de sintomas apresentam: dispneia, tosse seca, febre, dor de cabeça. Os casos nem sempre apresentam suas manifestações clínicas típicas, podendo os indivíduos apresentarem formas assintomáticas da doença, representando uma possível identificação da doença e interagindo de forma direta na curva de aumento da transmissão. (1)

Mesmo após um ano de instaurada a pandemia, a infecção pelo Coronavírus ainda se caracteriza como uma emergência de saúde pública. Segundo dados recentes da OMS, foram registrados em 12 de fevereiro de 2021 um total de 107.423.526 casos e 2.360.280 óbitos em todo o mundo.(2)

O período que corresponde da manifestação inicial dos sintomas ao óbito pode variar de 6 a 41 dias, dispondo de uma mediana de 14 dias, essa informação sinaliza que a detecção precoce de casos suspeitos representa uma importante janela de ação. 


\section{artigo}

Júnior, A. R.C., Martins, M. I. S., Alcântara, D. G., Santos, M. A. P., Teixeira, A. N. A., Gomes, T. F., Dores, C. C. C., Freitas, F. A. S., Porto, A. P. M., Moreira, F. J. F.. Investigação clínica e sintomatologica de pacientes acometidos pela covid-19 entre 2020 e 2021 no Ceará

O Brasil apresenta uma taxa de testagem inferior ao esperado, quando comparado aos demais países que visam o controle da pandemia, aliado a excussão ineficaz da detecção, a flexibilização de medidas de distanciamento social por pressões econômicas e políticas junto a instabilidades na coordenação setorial no campo da saúde pública enfraqueceram o combate desse agravo.(3)

As diferenças regionais, socioeconômicas, epidemiológicas e climáticas presentes no Brasil significam diferenças expressivas na relação de incidência e prevalência da infecção pelo SARS-CoV-2.(4) Vale destacar que nas capitais dos estados das regiões Norte e Nordeste, como Rio Branco (Acre), Natal (Rio Grande do Norte), Belém (Pará) e Fortaleza (Ceará), mais de $90 \%$ das unidades de terapia intensiva (UTIs) hospitalares já estavam ocupadas em meados de maio.(5)

Em países em desenvolvimento, como caso do Brasil, o processo de testagem em massa não foi efetivo, sendo priorizados pacientes em sintomas clássicos ou em internamento. A subnotificação dos casos dificultou o conhecimento sobre a real situação epidemiológica no país. (6)

A dimensão continental do país e a imensa demanda de insumos e profissionais necessários para o controle e disponibilidade de testes para uma maior cobertura populacional tornam o enfrentamento à COVID-19 um desafio ainda maior. Com isso, reforça-se a ideia do reconhecimento de sinais e sintomas clínicos apresentados pela população infectada contribuindo para o estabelecimento de fluxos de atendimento e priorização na realização de exames confirmatórios. (7) Diante do informado, objetivou-se descrever o comportamento clínico e sintomatológico de pacientes acometidos pela COVID-19 no Ceará.

\section{MÉTODO}

Trata-se de um estudo descritivo, retrospectivo, de análise documental com abordagem quantitativa, realizado em 3 hospitais de referência no estado do Ceará para tratamento da COVID-19, distribuídos nas Regiões de Saúde: Sertão Central,
Cariri e Região Norte. A pesquisa se insere em um projeto desenvolvido pela Escola de

Em países em

desenvolvimento,

como caso do

Brasil, o processo de

testagem em massa

não foi efetivo,

sendo priorizados

pacientes

em sintomas

clássicos ou em

internamento. A

subnotificação dos

casos dificultou

o conhecimento

sobre a real situação

epidemiológica no

país

Saúde Pública do Estado do Ceará-ESP, junto à Secretaria de Saúde do Estado do
Ceará-SESA, intitulado "ResCOVID: Desenvolvimento de um sistema de registro clínico eletrônico para pacientes hospitalizados com COVID-19 no Ceará/Brasil".

Para esse estudo, foram incluídos pacientes com internação por COVID-19, que possuíam teste diagnóstico RT-PCR detectável. Foram excluídos pacientes transferidos, justificando-se pela necessidade de acompanhamento do paciente até seu desfecho na hospitalização pela doença. Assim, o universo de pacientes coletados neste hospital compreende 1657. Após a aplicação dos critérios de inclusão e exclusão, a amostra de pacientes contou com 965 representantes. A coleta de informações ocorreu em prontuários eletrônicos disponíveis na sede das instituições compreendendo o período de maio de 2020 a outubro de 2021. A amostra abrange a divisão da coleta em dois momentos, constando de pacientes com data internação da primeira onda (março a maio de 2020), e segunda onda (janeiro a abril de 2021).

Considerando o desenvolvimento de um estudo descritivo, os dados foram apresentados conforme estatística descritiva com utilização de frequência absoluta e percentual. Foram descritas as variáveis: idade (representada por faixa etária), cor, escolaridade, atividade profissional, hábitos pessoais (tabagismo, consumo de bebida alcoólica), comorbidades e sintomas iniciais. Traçando assim um perfil de pacientes internados, além da descrição dos suportes utilizados para oxigenoterapia e desfechos.

As informações coletadas foram armazenadas em uma plataforma exclusiva intitulada "ResCOVID". Esta ferramenta facilitou o trabalho dos pesquisadores, deixando mais ágil o modelo de coleta e mais segura a forma de armazenamento, assim como o acesso às informações para consulta.

A pesquisa cumpre a todos os princípios éticos ao tratar com dados de pacientes, respeitando o anonimato e segurança de dados. O trabalho segue as determinações da Resolução 466 de 12 de dezembro de 2012, estando aprovado pelo Comitê de Ética em Pesquisa da Escola de Saúde Pública do Ceará, conforme CAAE 30423920.0.0000.5037. 


\section{RESULTADOS}

As características sociodemográficas dos 965 pacientes sintetizadas conforme tabela 1 aponta para a divisão entre $1^{\mathrm{a}} \mathrm{e} 2^{\mathrm{a}}$ onda de contaminação pela COVID-19 no Brasil. $\mathrm{Na}$ tabela é possível identificar o predomínio dos pacientes com faixa de idade $>60$ anos, $88(57,1 \%)$ e 416 (51\%), respectivamente conforme as ondas, seguido por pacientes entre 50 e 59 anos, $26(16,9)$ e 140 (17\%). Temos um predomínio de indivíduos do sexo masculino, apresentando 89 (57,8\%) e 454 (56\%), conforme onda.

Em relação à cor, pardos foram maioria em ambas as ondas, contando com 74 (48\%) e 382 (47\%). Quanto à escolaridade, a maioria dos pacientes declarantes relatou ensino fundamental incompleto, 47 (30,5\%) e 167 (20,6\%). Sobre a execução de atividades profissionais tem-se que na primeira onda um total de 37 (24\%) relata atividade não remunerada, enquanto na segunda onda temos o predomínio de 387 (47\%) de pacientes declarando atividade remunerada. Considerando os hábitos pessoais, $9(5,8 \%)$ e $52(6,4 \%)$ eram tabagistas; $15(9,7 \%)$ e $163(20 \%)$ pacientes eram ex-tabagistas, enquanto $10(6,5 \%)$ e $24(2,6 \%)$ eram etilistas, na primeira onda não houve relato sobre práticas de ex-tabagismo, enquanto na segunda $14(1,7 \%)$ se declararam ex-etilistas.

As características clínicas dos pacientes estão sintetizadas na tabela 2. Dentre as comorbidades relatadas no estudo houve um predomínio de pacientes nas duas ondas, hipertensão $75(48,7 \%)$ e 364 (44,9\%), seguido de pacientes com diabetes 38 (36,7\%) e 222 (27,4\%); doença cardíaca aparece na primeira onda como terceira colocada em número de casos com 19 (\%), enquanto fica em quarto na segunda onda apresentando 129 (15,9\%); obesidade apresenta-se em na primeira onda como quarta colocada em número de casos contando com $19(12,3 \%)$, na segunda onda apresentam 56 (6,9\%).

Os sintomas iniciais relatados com maior frequência considerando a distribuição entre primeira e segunda onda res-
Tabela 1: Características sociodemográficas de pacientes acometidos pela COVID-19 no Ceará, Brasil. 2021.

\section{Características sociodemográficas da população estudada ( $\mathrm{N}=965)$}

\begin{tabular}{|c|c|c|c|c|}
\hline \multirow{2}{*}{$\begin{array}{l}\text { Período } \\
\text { Variáveis }\end{array}$} & \multicolumn{2}{|c|}{ 1ª Onda (154) } & \multicolumn{2}{|c|}{$2^{\mathrm{a}}$ Onda (811) } \\
\hline & N & $\%$ & $\mathrm{~N}$ & $\%$ \\
\hline \multicolumn{5}{|l|}{ Idade (anos) } \\
\hline$<20$ & 5 & 3,2 & 14 & 1,7 \\
\hline $20-29$ & 5 & 3,2 & 39 & 4,8 \\
\hline 30-39 & 9 & 5,8 & 91 & 11,2 \\
\hline $40-49$ & 21 & 13,6 & 111 & 13,7 \\
\hline $50-59$ & 26 & 16,9 & 140 & 17 \\
\hline$\geq 60$ & 88 & 57,1 & 416 & 51 \\
\hline \multicolumn{5}{|l|}{ Sexo } \\
\hline M & 89 & 57,8 & 454 & 56 \\
\hline$F$ & 65 & 42,2 & 357 & 44 \\
\hline \multicolumn{5}{|l|}{ Cor } \\
\hline Pardo & 74 & 48 & 382 & 47 \\
\hline Branco & 23 & 14,9 & 113 & 14 \\
\hline Preto & 4 & 2,6 & 20 & 2,3 \\
\hline Não referido & 53 & 34,4 & 296 & 36,7 \\
\hline \multicolumn{5}{|l|}{ Escolaridade } \\
\hline Sem Escolaridade & 33 & 21,4 & 128 & 15,8 \\
\hline Ensino Fundamental Incompleto & 47 & 30,5 & 167 & 20,6 \\
\hline Ensino Fundamental Completo & 10 & 6,5 & 56 & 7 \\
\hline Ensino Médio Incompleto & 3 & 1,9 & 14 & 1,7 \\
\hline Ensino Médio Completo & 3 & 1,9 & 110 & 13,6 \\
\hline Ensino Superior Incompleto & 1 & 0,6 & 11 & 1,3 \\
\hline Ensino Superior Completo & 9 & 5,7 & 44 & 5,4 \\
\hline Não Informado & 48 & 31 & 281 & 34,6 \\
\hline \multicolumn{5}{|l|}{ Atividade Profissional } \\
\hline Remunerada & 25 & 16,2 & 387 & 47 \\
\hline Não Remunerada & 37 & 24 & 167 & 20,1 \\
\hline Não Informado & 92 & 59,7 & 257 & 32,9 \\
\hline \multicolumn{5}{|l|}{ Hábitos Pessoais } \\
\hline Tabagista & 9 & 5,8 & 52 & 6,4 \\
\hline Ex-Tabagista & 15 & 9,7 & 163 & 20 \\
\hline Etilista & 10 & 6,5 & 24 & 2,6 \\
\hline Ex-Etilista & 0 & - & 14 & 1,7 \\
\hline
\end{tabular}

Legenda: N (valor absoluto); \% (taxa em porcentagem); $1^{\mathrm{a} o n d a}$ (período que corresponde a março a maio de 2020); $2^{\mathrm{a}}$ onda (período que corresponde a janeiro a abril de 2021).

Fonte: Elaborado pelos autores.

pectivamente foram: dispneia com registro de $112(72,7 \%)$ e 610 (75,2\%); tosse 108
$(70 \%)$ e $491(55,7 \%)$; febre $104(67,5 \%)$ e 443 (50,2\%); mialgia $18(11,7 \%)$ e 157 


\section{artigo}

Júnior, A. R.C., Martins, M. I. S., Alcântara, D. G., Santos, M. A. P., Teixeira, A. N. A., Gomes, T. F., Dores, C. C. C., Freitas, F. A. S., Porto, A. P. M., Moreira, F. J. F. Investigação clínica e sintomatologica de pacientes acometidos pela covid-19 entre 2020 e 2021 no Ceará

(19,3\%); cefaleia $15(9,7 \%)$ e $116(14,3 \%)$ Outros sintomas também reportados com menor frequência, tais como dor de garganta $10(6,55 \%)$ e $77(9,5 \%)$; coriza $13(8,4 \%)$ e $55(6,8 \%)$ e astenia $8(5,2 \%)$ e $79(9,7 \%)$, dentre outros.

Os suportes respiratórios estão sintetizados na tabela 3. A utilização de suporte respiratório mais utilizado na primeira onda foi a ventilação mecânica invasiva com 58 $(37,7 \%)$, o mesmo suporte teve na segunda onda a incidência de 295 (36,8\%). A máscara reservatório representou 45 (29,2\%) na primeira onda, sendo maior destaque na segunda onda com 401 casos $(49,4 \%)$; seguido do uso de cateter nasal de baixo fluxo com respectivamente 39 (25,3\%) e 201 (24,8\%). A ventilação mecânica não invasiva teve uma grande variação, tendo apenas $2(1,3 \%)$ de casos na primeira onda, sendo $272(33,5 \%)$ dos casos na segunda onda.

Considerando a relação desfecho, os pacientes apresentam durante a primeira onda número de óbitos de $16(10,4 \%)$ e alta de $138(89,6 \%)$, enquanto na segunda onda
Tabela 2: Comorbidades e sintomas iniciais de pacientes acometidos pela COVID-19 no Ceará, Fortaleza-CE, Brasil. 2021.

\section{Características clínicas da população estudada $(n=965)$.}

\begin{tabular}{lcccc} 
Período & \multicolumn{2}{c}{$1^{\text {a }}$ Onda (154) } & \multicolumn{2}{c}{$2^{\text {a }}$ Onda (811) } \\
Variáveis & $\mathrm{N}$ & $\%$ & $\mathrm{~N}$ & $\%$ \\
Comorbidades & & & & \\
Hipertensão & 75 & 48,7 & 364 & 44,9 \\
Diabetes & 38 & 26,7 & 222 & 27,4 \\
Doença Cardíaca & 19 & 12,3 & 56 & 6,9 \\
Obesidade & 12 & 7,8 & 129 & 15,9 \\
DPOC & 12 & 7,8 & 36 & 4,4 \\
Doença Neurológica & 9 & 5,8 & 50 & 6,2 \\
Asma & 8 & 5,2 & 15 & 1,9 \\
Doença Renal Crônica & 5 & 3,2 & 17 & 2 \\
Doença Reumatológica & 2 & 1,3 & 11 & 1,4 \\
Doença Autoimune & 2 & 1,3 & 10 & 1,2 \\
Doença Hepática Crônica & 2 & 1,3 & 8 & 0,9
\end{tabular}

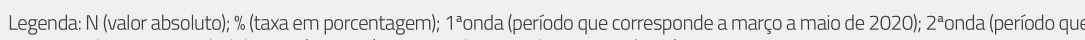
corresponde a janeiro a abril de 2021); DPOC (Doença Pulmonar Obstrutiva Crônica).

Fonte: Elaborado pelos autores.

\section{Gráfico 1: Distribuição percentual de sintomas de pacientes acometidos pela COVID-19 no Ceará, organizados por onda,} Fortaleza, CE, 2021.

100

90

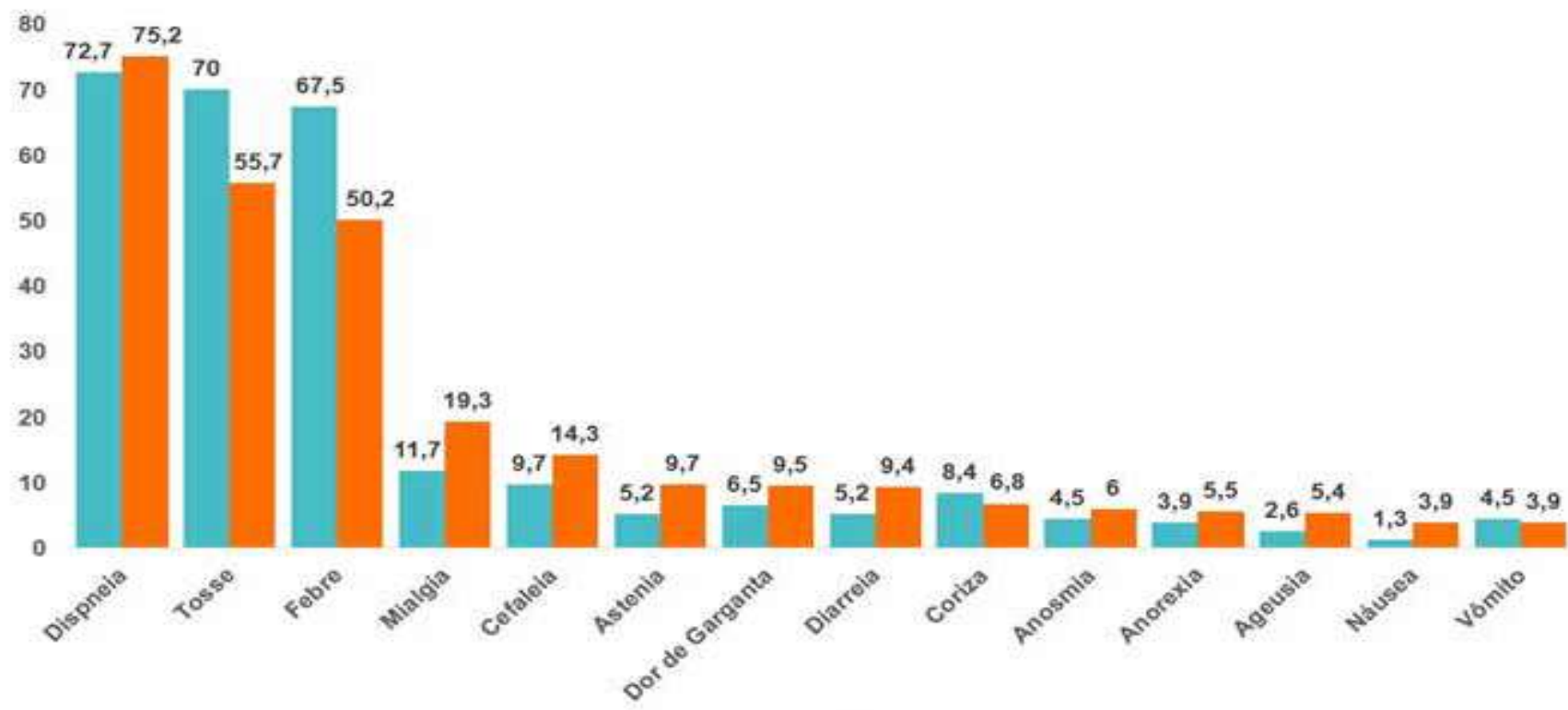

Fonte: Elaborado pelos autores. 
número de óbitos.

\section{DISCUSSÃO}

Em correspondência aos dados expostos no presente artigo, o perfil de pacientes mais acometidos pela COVID-19 tanto na primeira onda, quando na segunda têm apresentado idades superior a 60 anos, corroborando com outras pesquisas, $(7,3)$ que demonstram ser esta faixa etária a mais atingida por casos de estados graves a críticos, estar associada a presença de um maior número de sintomas e sintomas mais graves.

Ressalta-se ainda, durante a segunda onda, um crescimento no acometimento das demais faixas de idade, com destaque às das pessoas de 30 a 39 anos, ganhando maior evidência a crescente infecção e número de óbitos entre os jovens devido à presença de novas cepas da doença e lentidão do processo de imunização, não conseguindo contemplar no início da segunda onda as faixas etárias menores.

Manteve-se ainda como mais prevalente o sexo masculino, coadunando com o perfil que é descrito na maioria das pesquisas, como na realizada no estado da Pararaíba, (8) que apresenta maior prevalência de infecção entre os homens com idade entre 50 e 70 anos; e mesmo nas pesquisas em que a prevalência foi maior entre as mulheres, observou maior gravidade dos casos com evolução para óbito entre os homens. (9)

Corroborando com os achados do presente artigo, outros autores também referem maior prevalência da cor parda em pacientes acometidos pela COVID-19. Em estudo realizado sobre as características epidemiológicas de contaminação no estado da Bahia (10) os autores constataram que $50,6 \%$ são da cor parda, $12,2 \%$ brancos e $8,2 \%$ pretos. Além dos pacientes acometidos, a cor parda foi predominante no número de óbitos, representando $55,1 \%$ dos casos.

Quanto à influência do nível de escolaridade, estudos apontam a necessidade de se considerar a relação direta desta com a renda familiar e a dificuldade ao acesso dos serviços de saúde. Evidenciou-se que pessoas com nível de escolaridade superior

Tabela 3: Suportes respiratórios de pacientes acometidos pela COVID-19 no Ceará, Fortaleza-CE, Brasil. 2021.

Suporte respiratório utilizado durante a internação da população estudada ( $n=965)$.

Período

Variáveis

Ventilação mecânica invasiva

Máscara com reservatório

Cateter nasal (baixo fluxo)

Ventilação mecânica não invasiva $1^{\text {a }}$ Onda (154)

N $\%$

58

45

37,7
$2^{\mathrm{a}}$ Onda (811)

Legenda: $\mathrm{N}$ (valor absoluto); \% (taxa em porcentagem); $1^{1}$ anda (período que corresponde a março a maio de 2020); $2^{2}$ onda (período
referente a janeiro a abril de 2021). referente a janeiro a abril de 2021)

Fonte: Elaborado pelos autores.

com caso grave da COVID-19 obtiveram melhora clínica e, consequentemente, uma menor proporção de óbitos (22,5\%), comparadas com pessoas sem escolaridade $(71,3 \%)$. Constata-se ainda que a escolaridade associada a cor potencializa o desfecho desses pacientes, em que pretos e pardos sem escolaridade apresentaram um número de mortes 4 vezes maior do que brancos com nível superior $(80,35 \%$ contra 19,65\%). (11)

Outro fator de risco tanto para a taxa de contaminação quanto para casos de maior gravidade da doença seria o tabagismo, devido a prática em si do ato de fumar (levando o produto à boca sem a higienização adequada das mãos, e em alguns casos o compartilhamento com outras pessoas do produto, como no caso dos cigarros eletrônicos e narguilés) e piora do comprometimento pulmonar, pelas alterações prévias fisiologicamente instaladas pelo uso crônico do tabaco, respectivamente.(12)

A pandemia representou alterações em outros campos sociais como o de trabalho, em que a execução das atividades laborais foi modificada, na maioria dos casos houve a migração para atividades de forma remota,(13) outras, pela característica essencial a população (nos campos da saúde, alimentação, transporte, outros), houve maior intensificação das jornadas de trabalho, sobretudo dos profissionais de saúde. $(13,14)$

Acerca da diferença de infecção entre as classes trabalhadoras na primeira e segun$\mathrm{da}$ onda, alguns fatores aos quais se pode associar tal condição seria a diminuição do medo do contágio e suas consequências com a chegada da vacina e a ideia ilusória de garantia da imunidade ao primeiro contágio, diminuição, ao final de 2020, dos casos infectados e ao avanço dos planos econômicos de retomada de atividade laboral. O que pode justificar a maior infecção entre a população que retorna ao ambiente de trabalho, mas antes, durante a primeira onda, podiam realizar atividades remotas, diferentemente da classe de trabalhadores autônomos, sem atividade remunerada.(15)

As comorbidades associadas também determinam maior predisposição à infecção e agravamentos da COVID-19. Assim como a prevalência do presente estudo, a hipertensão lidera como a comorbidade com maior incidência de comprometimento na doença.(11) Apontou-se no presente estudo a maior incidência da obesidade na segunda onda da pandemia em comparação com a primeira. Salienta-se que a obesidade é considerada um fator agravante para doenças respiratórias e promove complicações adversas, como baixa saturação pela dificuldade de ventilação pulmonar, produção de secreções anormais de adipocinas e citocinas, acúmulo de tecido adiposo que pode servir de reservatório do adenovírus humano, além do alto risco de eventos trombóticos e desenvolvimento de outras doenças crônicas, aumentando assim as chances de óbito.(16)

Os sinais e sintomas na grande maioria das vezes funciona como sistema sensível e à predição de necessidade de atendimento médico-hospitalar, bem como exames complementares necessários à tomada de decisão para intervenção.(7) 


\section{artigo}

Júnior, A. R.C., Martins, M. I. S., Alcântara, D. G., Santos, M. A. P., Teixeira, A. N. A., Gomes, T. F., Dores, C. C. C., Freitas, F. A. S., Porto, A. P. M., Moreira, F. J. F.. Investigação clínica e sintomatologica de pacientes acometidos pela covid-19 entre 2020 e 2021 no Ceará

Dos sintomas mais presentes nas duas ondas da COVID-19 no Ceará podem-se citar a dispneia, tosse e febre, enquanto para todos os sintomas apresentados houve um aumento da frequência na segunda onda, para a qual aponta-se principalmente um crescimento nos sintomas referidos de mialgia, cefaleia, astenia, dor de garganta (odinofagia) e diarreia.

Em sua maioria, os sintomas mais comumente apresentados pelos pacientes infectados pela COVID-19 são dispneia, tosse, febre e mialgia, corroborando aos resultados apresentados neste estudo. Sintomas gastrointestinais, como a diarreia, anorexia e vômitos, podem fazer parte do quadro clínico de 3\% a 79\% dos pacientes, fazendo-se mais frequente, sobretudo, nos casos mais graves ou entre a população pediátrica e de adolescentes, devendo-se chamar atenção para os riscos associados de desnutrição e sarcopenia.(17)

$\mathrm{Na}$ investigação realizada com pacientes positivos para a COVID-19 na China, 98\% apresentaram febre, $76 \%$ tosse, $55 \%$ dispneia e $44 \%$ mialgia/fadiga, sendo os sintomas menos comuns como a anosmia/ hiposmia e ageusia, na ausência de doenças respiratórias alérgicas prévias, os sintomas mais sugestivos da doença, isto é, de casos suspeitos mais sensíveis à doença, antes do diagnóstico laboratorial. Sendo que a maior atenção dada a determinados sintomas, em especial aos gripais no geral, foi decorrente da tentativa de orientar quanto a necessidade do isolamento social e, por conseguinte, da diminuição da transmissão viral.(7)

Em estudo realizado acerca dos sintomas, observou-se que alguns fatores estiveram relacionados a maiores chances de apresentar todos os sintomas e maior prevalência dos mesmos, tais características foram de indivíduos: do sexo feminino, com maior escolaridade, idades mais avançadas, e que fossem das regiões norte e nordeste. (18) As duas primeiras podem ser associadas a maior facilidade e detalhamento em descrever os sintomas apresentados e as duas últimas relacionadas à maior vulnerabilidade orgânica ou socioeconômica para a infecção, respectivamente.

Alguns pacientes podem evoluir para a forma grave da doença caracterizada como Síndrome Respiratória Aguda Grave (SRAG), a qual é determinada pela evolução da dispneia persistente, baixa saturação de oxigênio (menor que 95\%) sem suporte de oxigênio ou áreas centrais com coloração azulada (lábios e face).(19)

Nos casos com necessidade de internação a suplementação de oxigênio está entre os fatores mais condicionantes desta deci-

\section{Em sua maioria,}

$$
\text { os sintomas mais }
$$

comumente

\section{apresentados pelos}

pacientes infectados

pela COVID-19

são dispneia, tosse,

febre e mialgia,

corroborando

aos resultados

\section{apresentados neste}

\section{estudo}

são. Dentre as modalidades terapêuticas a Ventilação Mecânica (VM) é essencial aos casos de insuficiência respiratória que requerem um nível de oxigenação adequado e descanso da musculatura respiratória. Pondera-se quanto ao uso da Ventilação Não Invasiva (VNI) o conhecimento clínico e expertise dos profissionais da saúde, na determinação dos casos que realmente são elegíveis ao uso da mesma, expondo-se pesquisas que encontraram índices elevados de erros em sua administração, chegando a $70 \% .(20,21)$
A apresentação da VNI com parâmetros diferenciados e sua utilização precoce estiveram correlacionados a menor necessidade de intubação orotraqueal, principalmente as realizadas através de capacetes (helmet), haja vista que a maioria dos estudos demonstrou alta taxa de mortalidade entre os pacientes que precisaram fazer uso de VM.(22) O que fundamenta a maior utilização de VNI na segunda onda, em decorrência do uso do sistema de interface do tipo capacete para aplicação contínua de pressão positiva.(23, 24)

Associado ao uso destes novos dispositivos de VNI, a implementação dos protocolos de pronação (para evitar o início ou prolongamento de medidas invasivas) e o início somado ao avanço da vacinação podem estar concatenadas à diminuição de óbitos apresentados na segunda onda analisada. Em que mesmo com a aplicação apenas de uma dose da vacina já há a diminuição de sintomas graves e óbitos entres os imunizados parcialmente. $(22,25)$

\section{CONCLUSÕES}

Os dados analisados e apresentados neste estudo permitem apresentar o perfil descritivo de pacientes acometidos pela COVID-19 no Ceará, descrevendo características populacionais assim como a observância sobre fatores clínicos e sintomatológicos. A amostra analisada coaduna com a literatura sobre a questão de perfil sintomatológico e clínico, evidenciando sintomas típicos com variações entre primeira e segunda onda.

Um importante achado para sinalizar é a diminuição significativa da proporção de óbitos geral, podendo estar relacionada desde as características das novas variantes presentes na segunda onda, como a possibilidade de testagem realizada com maior brevidade, assim como o reconhecimento dos sintomas de forma mais clara. Desse modo, o estudo sinaliza para a necessidade de continuidade nas investigações, sobretudo pensando na verificação sobre a gravidade dos sintomas e sua relação com o desfecho. 


\section{REFERÊNCIAS}

1. Zhu N, Zhang D, Wang W, Li X, Yang B, Song J, et al. A novel coronavirus from patients with pneumonia in China, 2019. N Engl J Med. [internet] 2020 [cited 2021 Set 20];382:727-33. DOI: http://dx.doi.org/10.1056/NEJMoa2001017

2. Saraiva, E.L.; Siqueira, M.E.B.; Sousa, C.S.;Assistência de enfermagem prestada ao paciente crítico com COVID-19: um relato de caso. Saúde Coletiva. [internet] 2021 [cited 2021 Out 25];11(esp): 6993-6999. DOI: https://doi.org/10.36489/saudecoletiva.2021v11iCOVIDp6993-7006

3. Moreira RS, Latent class analysis of COVID-19 symptoms in Brazil: results of the PNAD-COVID19 survey. Cad. Saúde Pública. [internet] 2021 [cited 2021 Set 20];37(1):e00238420 DOl: http://dx.doi.org/10.1590/0102-311X00238420

4. Brazil Coronavirus map and case count. The New York Times. [internet] 2020 [cited 2021 Set 20]. https://www.nytimes. com/ interactive/2020/world/americas/bra zil-coronavirus-cases. html (acessado em 22/ Jun/2020).

5. Macinko J, et al., Health care seeking due to COVID-19 related symptoms and health care cancellations among older Brazilian adults: the ELSI-COVID-19 initiative. Cad. Saúde Pública. [internet] 2020 [cited 2021 Set 20] ;i 36 Sup 3:e00181920. DOI: http:// dx.doi.org/10.1590/0102-311X00181920

6. Accorsi TA, De Amicis K, Brígido AR, Belfort DS, aniHabrum FC, Scarpanti FG, et al. Avaliação de pacientes com sintomas respiratórios agudos durante a pandemia de COVID-19 via Telemedicina: características clínicas e impacto no encaminhamento. Einstein (São Paulo). [internet] 2020 [cited 2021 Set 20];18:eA06106. DOI: http://dx.doi.org/10.31744/einstein_ journal/2020A06106

7. Iser BPM, et al., Suspected COVID-19 case definition: a narrative review of the most frequent signs and symptoms among confirmed cases. Epidemiol. Serv. Saude, Brasilia. [internet] 2020 [cited 2021 Set 20]; 29(3):e2020233. DOI: http://dx.doi. org/10.5123/S1679-49742020000300018

8. Medeiros LCA, Borges MCAL, Gonsalves MP, Teodózio GC, SAntos WMI. Perfil sócio demográfico dos pacientes acometidos pela COVID-19. Environmental Smoke. [internet] 2021 [cited 2021 Set 21]; 4 (2): 42-48. DOI: http://dx.doi.org/10.5380/atoz. v9i2.76179

9. Leite TGG, Mafra SC, Araújo RF dos A, Silva EM de OM, Cardoso $A M$ de J. Entendimento e adesão dos jovens às medidas de restrição social na pandemia de COVID-19. REAS [Internet]. 2021 [cited 2021 Set 21];13(7):e7692. DOI: https://doi.org/10.25248/ reas.e7692.2021

10. Machado AG, Batista MS, Souza M de C. Características epidemiológicas da contaminação por COVID-19 no estado da Bahia. Rev. Enferm. Contemp., Salvador. [internet]. 2021 [cited 2021 Set 21];10(1):103-110. DOl:https://doi.org/10.17267/23173378rec.v10i1.3594

11. Batista A, Antunes B, Faveret G, Peres I, Marchesi J, Cunha JP, et al. Análise socioeconômica da taxa de letalidade da COVID-19 no Brasil. Nucleo de Operacoes e Inteligencia em Saúde (NOIS). [internet] 2020 [cited 2021 Set 21]; Disponivel em: https://www. sites.google.com/view/nois-pucrio/publica\%C3\%A7\%C3\%B5es?authuser=0\#h.ijqa5msfttoq

12. Borges GM, Crespo CD. Aspectos demográficos e socioeconômicos dos adultos brasileiros e a COVID-19: uma análise dos grupos de risco a partir da Pesquisa Nacional de Saúde, 2013. Cad. Saúde Pública. [internet] 2020 [cited 2021 Set 21];36(10):e00141020. DOI: https://doi.org/10.1590/0102$311 \times 00141020$

13. Almeida WS, Szwarcwald CL, Malta DC, Barros MBA, Souza Júnior PRB, Azevedo LA, et al. Mudanças nas condições socioeconômicas e de saúde dos brasileiros durante a pandemia de covid-19. Rev Brasileira de Epid. [internet] 2020 [cited 2021 Set 21];23(1):1-30. doi: https://doi.org/10.1590/SciELOPreprints. 1272

14. Vedovato TG, Andrade CB, Santos DL, Bitencourt SM, Almeida LPD, Sampaio JFDS. Trabalhadores (as) da saúde e a COVID-19: condições de trabalho à deriva?. Rev Bras Saude Ocup. [internet] 2021 [cited 2021 Set 23];46:(esp)1:1-15. DOI: https://doi. org/10.1590/2317-6369000028520

15. Almeida SM, Andrade CAS de, Castro JDSM, Almeida CS, Almeida AC de. Perfil epidemiológico dos casos de Covid-19 relacionados ao trabalho no estado da Bahia. Revista Baiana de Saúde Pública. [internet] 2021 [cited 2021 Set 25];45(Esp):93108. DOI: https://doi.org/10.22278/2318-2660.2021.v45.NEspecial_1.a3248

16. Costa TRM, Correia RS, Silva PH dos S, Barbosa GSL, Oliveira LM de, Cruz VT de et al. A obesidade como coeficiente no agravamento de pacientes acometidos por COVID-19. Research, Society and Development. [internet] 2020 [cited 2021 Set 23];9(9): e395997304-e395997304. DOI: http://dx.doi.org/10.33448/ rsd-v9i9.7304

17. Oba J, Carvalho WB de, Silva AC, Delgado AF. Sintomas gastrintestinais e abordagem nutricional durante a pandemia de COVID-19: guia prático para pediatras. Einstein (São Paulo). [internet] 2020 [cited 2021 Set 23];18:1-8. DOI: http://dx.doi. org/10.31744/einstein_journal/2020RW5774

18. Moreira R da S. Análises de classes latentes dos sintomas relacionados à COVID-19 no Brasil: resultados da PNADCOVID19. Cad. Saúde Pública. [internet] 2021 [cited 2021 Out 05];37(1):e00238420. DOI: https://doi.org/10.1590/0102$311 \times 00238420$

19. Santo JF, Santos DD. Hierarquia de sintomas de Gripe relacionados à COVID-19 de acordo com sexo e cor ou raça em notificações de pacientes com Síndrome Respiratória Aguda Grave no Brasil. [internet] 2021 [cited 2021 Out 05];25(06):1-15 DOI: https://doi.org/10.1590/SciELOPreprints.1161

20. Carrillo-Esper R, Mejía-Gómez L, Monares-Zepeda E, Chavarría-Martínez U, Díaz-Carrillo A, Ayala-León M et al. Abordaje hemodinámico y ventilatorio en pacientes con COVID-19. Cirugía y Cirujanos. [internet] 2020 [cited 2021 Out 05];88(6):805-817. DOI: https://doi.org/10.24875/ciru.20000301 


\section{artigo}

Júnior, A. R.C., Martins, M. I. S., Alcântara, D. G., Santos, M. A. P., Teixeira, A. N. A., Gomes, T. F., Dores, C. C. C., Freitas, F. A. S., Porto, A. P. M., Moreira, F. J. F.. Investigação clínica e sintomatologica de pacientes acometidos pela covid-19 entre 2020 e 2021 no Ceará

\section{REFERÊNCIAS}

21. Cruz DA, Sousa I de L, Santana PVD, Oliveira LK de A, Sousa FW dos S, Araújo AMX de. Impactos da ventilação mecânica invasiva em pacientes de COVID-19: revisão integrativa. Research, Society and Development. [internet] 2021 [cited 2021 Out 05];10(11):e380101119656-e380101119656. DOI: 10.33448/ rsd-v10i11.19656.

22. Botelho LL, Sebim Álisson FC, Paula ACAL de, Gomes A de M, Filho ILC, Santos JR, Matta MLB da, Furtado MC, Bastos PPB, Sales TM. Ventilação mecânica, parâmetros de troca gasosa e desmame do ventilador em pacientes com COVID-19. REAC [internet] 2021 [cited 2021 Out 06];28:e7914. DOI: https://doi. org/10.25248/reac.e7914.2021

23. Holanda MA, Tomaz BS, Menezes DGA de, Lino JA, Gomes GC. Desenvolvimento de um capacete para oferta de CPAP e oxige- noterapia com alto fluxo: ELMO 1.0. J Bras Pneumol. [internet] 2021 [cited 2021 Out 06];47(3):e20200590. DOl: https://dx.doi. org/10.36416/1806-3756/e20200590

24. Quadros TCC, Horn TL, Ribas A, Blattner C, Soares BP, Melo DA da S. Utilização da posição prona em ventilação espontânea em paciente com COVID-19. Scientia Medica Porto Alegre. [internet] 2021 [cited 2021 Out 06];31:1-8 e-39982. Avaliable from: https://revistaseletronicas.pucrs.br/ojs/index.php/scientiamedica/article/view/39982/26682

25. Lorenz C, Ferreira PM, Masuda ET, Lucas PC de C, Palasio RGS, Nielsen $L$ et al. COVID-19 no estado de São Paulo: a evolução de uma pandemia. Rev Bras Epidemiol. [internet] 2021 [cited 2021 Out 10];24: (Esp210040):1-14. DOI: https://doi. org/10.1590/1980-549720210040 